XVIII.

(Aus dem pathologischen Institut der Universität München. Direktor: Prof. Max Borst.)

\title{
Ueber Transplantationen experimentell erzeugter atypischer Epithelwucherungen.
}

Von

Kurt Freiherrn von Lamezan.

(Mit 3 Textfiguren.)

Bernlard Fischer ${ }^{1}$ ) ist es bekanntlich zuerst gelungen, atypische Epithel wucherungen durch subliutane Injektion von Scharlachrot und Sudan III in öliger Lösung am Kaninchenohr experimentell zu erzeugen. Diese Versuche wurden von einer Reihe von Autoren nachgeprift und bestïtigt, und dabei die Reihe der Stoffe, mit denen die Erzeugung atypischer Epithelwucherungen gelingt, um ein Bedeutendes erweitert. Durch Wacker und Schmincke2), welche eine grosse Anzahl von den verschiedenartigsten Stoffen zu ihren Versuchen verwandten, konnte gezeigt werden, dass es die Lipoidlöslichkeit der angewandten Stoffe ist, welche als der auslösende Faktor für die Erzeugung der Epithelwucherungen anzusehen ist. Nur diejenigen Stoffe haben die Fähigkeit, die Epithelien zu einer atypischen Wucherung anzuregen, welche in der Lage sind, wegen ihrer Lipoidlöslichkeit die lipoiden Zellhüllen in gewisser Weise zu verändern. Den beiden Autoren ist es gelungen, durch subkutane Injektion von Tabaksteer, Rohparaffinöl, Glanzrussextrakt am Kaninchenohr Wucherungen zu erzeugen, welche in ihrer Morphologie sowie in der Art ihres Wachstums eine unverkennbare Aehnlichkeit mit dem carcinomatösen Wachstum aufwiesen. Von Interesse ist dieses Resultat dadurch, dass diese Stoffe solche sind, welche vielleicht in der Aetiologie der sogenannten Berulscarcinome eine gewisse Rolle spielen.

Die wichtigste Frage bei den experimentell erzeugten atypischen Epithelwucherungen ist die, ob es sich nur um eine äussere Aehnlichkeit der-

1) B. Fischer, Experimentelle Erzeugung atypischer Epithelwucherungen und die Entstehung bösartiger Geschwülste. Münch. med. Wochenschr. 1906.

2) L. Wacker und A. Schmincke, Experimentelle Untersuchungen zur kausalen Genese atypischer Epithelwucherungen. Münch. med. Wochenschr. 1911. 
selben mit dem Carcinom handelt, oder ob eine innere und auch biologisch sich zeigende Wesensverwandtschaft zwischen beiden besteht. Die Möglichkeit, diese Frage einer Klärung entgegenzuführen, schien gegeben durch die Untersuchung ihres Verhaltens bei Transplantationen. Ueber derartige Untersuchungen von Transplantationen, teils subkutanen, teils intravenösen will ich in folgendem berichten:

Ich stïtzte mich dabei auf Versuche, welche von den Herren Prof. Schmincke und Dr. Wacker, teils von mir selbst unternommen worden sind. Ich gestatte mir, auch an dieser Stelle sowohl den beiden genannten Herren für die Ueberlassung des Materials und die vielseitige Unterstïtzung als auch Herrn Prof. Borst für das rege Interesse, das er meiner Arbeit entgegenbrachte, meinen verbindlichsten Dank auszusprechen.

Die atypischen Epithelwucherungen, die ich zu meinen Versuchen verwandte, erzeugte ich nach Angabe von Wacker und Schmincke in folgender Weise:

1. Rohparaffinöl wurde mit Wasserdampf destilliert und mit dem Destillat eine 10 proz. Lösung in Kaninchenfett hergestellt.

2. Es wurde der Inhalt einer verteerten Tabakspfeife mit Alkohol ausgezogen und der nach Verdampfung des Alkohols gewonnene Tabaksaft mit Kaninchenfett oder Olivenöl versetzt.

Diese Stoffe wurden mittels einer Rekordspritze den Versuchstieren subkutan ins Ohr injiziert.

Als Kontrollversuche dienten Transplantationen von normalem Epithel.

\section{Transplantationen experimentell erzengter atypischer Epithel- wucherungen unter den Panniculus carnosus.}

Subkutane Transplantationen experimentell erzeugter atypischer Epithelwucherungen sind bis jetzt nur von Huguénin ${ }^{1}$ ) vorgenommen worden. Dieser Autor transplantierte kleine Stückchen von Epithelwucherungen, die er durch Injelition von Scharlachrot in öliger Lösung am Kaninchenohr erzeugt hatte, demselben Tiere (autoplastisch) an eine andere Stelle des Körpers (die genaue Einpflanzungsstelle ist nicht angegeben). Sämtliche Gewebsstücke wurden resorbiert. Bei meinen Versuchen wurden Stückchen von den Epithelwucherungen vom Kaninchenohr in das Bindegewebe unter dem Panniculus carnosus der Bauchhaut, teils demselben Tiere (autoplastisch), teils einem anderen Tiere (homoioplastisch) transplantiert. 'In einigen Versuchen wurde das autoplastisch einige Zeit gewucherte Transplantat auf ein anderes Tier homoioplastisch weiter verpflanzt. Diese Versuchsanordnung wurde gewählt in der Meinung, es könnte bei wiederholten Verpflanzungen eine Steigerung der Wachstumstendenz des Epithels eintreten in derselben Weise, wie dies

1) Huguénin, Arch. de méd. éxpérim. et anat. path. 1910. p. 412. 
bei ähnlicher Versuchsanordnung bei Transplantationen von Tiergeschwülsten - Carcinomen und Sarkomen - beobachtet worden ist.

Die Versuchsanordnung gestaltete sich so, dass kleine Stückchen gewucherten sowohl wie normalen Epithels des Ohres nach sorglaltigem Rasieren der Ohrhaut und Abwaschen derselben mit Aether und Alkohol mit dem Rasiermesser abgetragen wurden und in vurher angelegte Taschen unter den Panniculus carnosus der Bauchhaut verpflanzt wurden. Von da wurden die Stückchen teils autoplastisch, teils homoioplastisch gewachsenen Epithels herausgeschnitten und teils mikroskopiert, teils homoioplastisch oder autoplastisch weiterverpflanzt.

\section{Autoplastik.}

Von gewuchertem Epithel.

Versuch $1 \mathrm{a}$ exstirpiert nach 8 Tagen. $\eta 1 \mathrm{~b}$ (vorher an "einer andern
Stelle 13 Tagen autoplast.
gewachsen.)

Versuch 2 exstirpiert nach 9 Tagen.

\section{Von normalem Epithel}

Kontrollversuche:

Versuch 1 exstirpiert nach 8 Tagen. (2 Versuche)

Versuch 2 exstirpiert nach 10 Tagen.

$\begin{array}{llllll}n & 3 & n & n & 22 & n \\ n & 4 & n & n & 26 & n \\ n & 5 & n & n & 38 & n\end{array}$

$\begin{array}{llllll}n & 3 & n & n & 10 & n \\ n & 4 & n & n & 14 & n \\ n & 5 & n & n & 16 & n \\ n & 6 & n & n & 38 & n\end{array}$

II. Homoioplastik.

Von gewuchertem Epithel.

Von normalem Epithel. Kontrollversuche:

Versuch 1 exstirpiert nach 8 T'agen.

$$
\begin{aligned}
& \begin{array}{lllll}
2 & & & 14 & 14
\end{array} \\
& \text { " } 3 \text { (vorher } 10 \mathrm{Tag}^{n} \text { autoplast. } \\
& \text { gewachsen.) }
\end{aligned}
$$

\section{Autoplastische Transplantationen von gewuchertem Epithel.}

Versuch 1. Rohparaftinölwucherung. Nach 8 Tagen wurde ein Stück exstirpiert, ein anderes Stück wurde an eine andere Stelle unter den Panniculus carnosus verpflanzt und nach 13 Tagen (also nach 21 tägigem Wachstum im ganzen) exstirpiert.

a) Das nach 8T'agen exstirpierte autoplastisch implantierte Stück gewucherten Epithels erweist sich als cystischer Hohlraum in Bindegewebe, welcher mit einem geschichteten verhornten Epitbel ausgekleidet ist. Im Lumen der Cysto findet sich horniges, lamellös geschichtetes, mit Leukozyten durchsetztes Material, Haarfragmente, sowie in Nekrose begriffene Lagen von Plattenepithel.

b) Das nach 8 Tagen exstirpierte und an eine andere Stelle unter den Panniculus carnosus replantierte und von hier nach 13 Tagen entfernte Epithelstück 
zeigt eine kugelige Epithelcyste. Die Wandung der Cyste wird durch geschichteles verhorntes Epithel gebildet. Das Lumen der Cyste ist mit lamellös geschichtetem, hornigem Material erfült. Das Epithei sitzt dem darunter gelegenen Bindegewebo glatt auf. Nirgends zeigen sich Zeichen einer stärkeren Proliferationstendenz des Epithels und Einwachsen des Epithels in das Bindegewebe. Im Bindegewebe findet sich viel Hämosiderinpigment.

Versuch 2. Rohparaffinölwucherung. Exstirpiert nach 9 Tagen. Das Präparat zeigt einen unterhalb des Panniculus carnosus gelegenen eystösen Hohlraum, dessen Inhalt von einer mit Leuliozyten durchsetzten Detritusmasso gebildet wird. In der äusseren Schicht dieses Detritus sind Haarfragmente mit anhängen-

Figur 1:

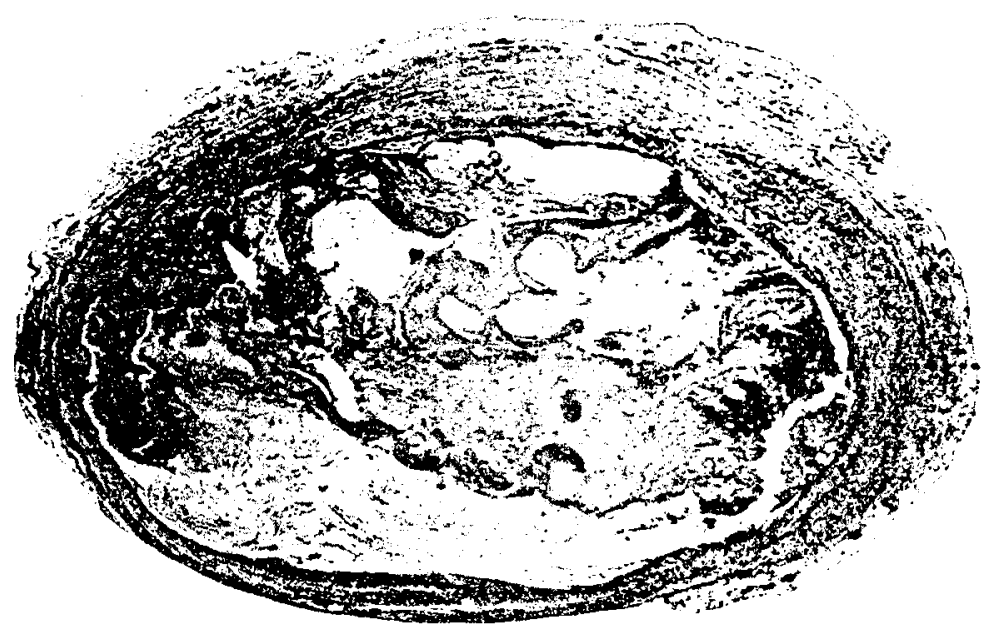

Subkutanc autoplastische Transplantation von gewuchertem Epithel; exstirpiert nach 14 Tagen. Vergrösserung 28 fach. (Versuch 4.)

der innerer Wurzelscheide erkennbar. Die Begrenzung des Hohlraums wird durch ein mehrschichtiges Plattenepithel gebildet, dessen innere, dem Lumen zngekebrte Schicht Verbornung aufweist. Das Epithel zeigt gute Kernfärbung. Von ihm aus gehen plumpe Sprossen und Zapfen in das benachbarte Bindegewebe. In einzelnen dieser in das benachbarte Bindegewebe gewucherten Epithelzapfen finden sich konzentrisch geschichtete Hornmassen. In der dem Bindegewebe aufsitzenden Fusszellenschicht, sowie in den in das Bindegewebe hineinragenden Epithelzapfen finden sich Mitosen in reichlicher Menge. Das umliegende Gewebe zeigt starke zellige Infijtration, und es findet sich hier teils frei, teils in Zellen eingeschlossen reichlich Hämosiderin-Pigment.

Versuch 3. Rohparaffinölwucherung. Exstirpiert nach 10 Tagen. Das Präparat zeigt einen Hohlraum im Bindegewebe. An einzelnen Stellen wird dieser Hohlraum ausgekleidet von einem Belag geschichteten Plattenepithels, welches oberflächliche Verhornung erkennen lässt. Als Inhaltsmasse des Hohlraums 
findet sich lamellös geschichtetes horniges Material, nicht weiter differenzierbarer Detritus, sowie Haare und Lagen von in Nelroso befindlichem Plattenepithel. Das umgebende Bindegewebe ist stark durchblutet und entzündlich zellig infiltriert.

Versuch 4. Tabaksteerwucherung, exstirpiert nach 14 T'agen. Im Bindegewebe findet sich ein kugeliger, mit geschichtetem, verhorntem Plattenepithel ausgekleideter Hohlraum. Als Inhaltsmasse finden sich Hornlamollen, zelliger Detritus und reichlich Haarfragmente mit anhängender Wurzelscheide. Das Epithel sitzt dem Bindegewebe glatt auf.

Figur 2,

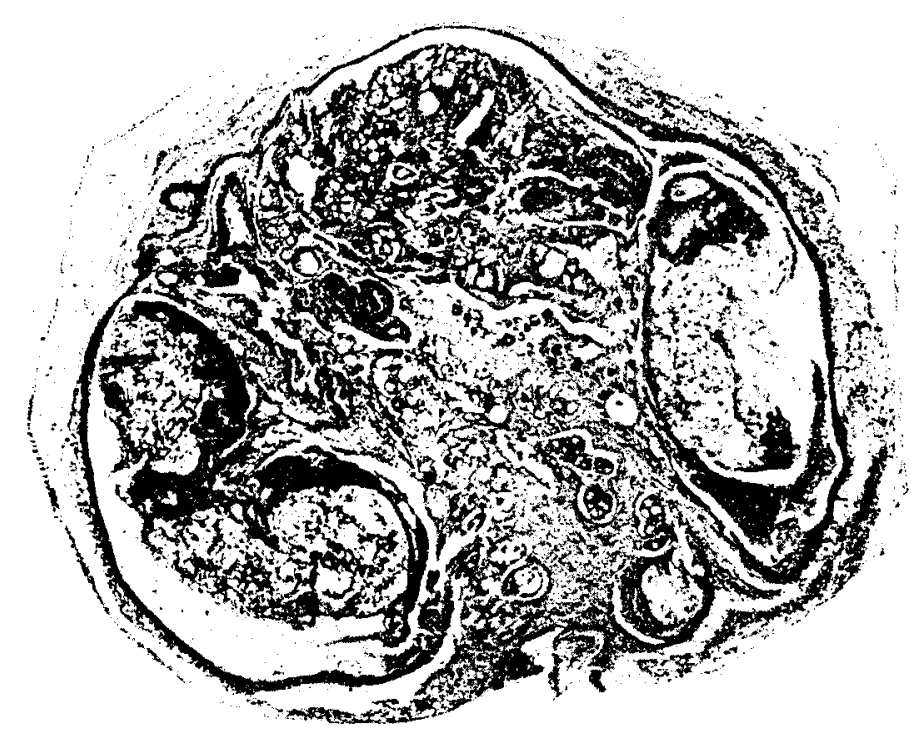

Subkutane autoplastische Transplantation von gewuchertem Epithel; exstirpiert nach 38 Tagen. Vergrösserung 30 fach. (Versuch 6.)

Versuch 5. Rohparaffinölwucherung, exstirpiert dem spontan verstorbenen Tiere nach 16 tägigem Wachstum. Das Präparat ist linsengross und erweist sich bei der mikroskopischen Untersuchung als eine mit verhorntem Plattenepithel ausgekleidete Cyste, deren Wandung jedoch nicht glatt, sondern in Falten gelegt ist. Als Inhaltsmasse finden sich Hornmassen, zerfallene Leukozyten und Haarfragmente.

Versuch 6. Rohparaffinölwucherung, exstirpiert nach 38 Tagen. Es findet sich eine klein-erbsengrosse Epithelcyste. Ihre Wandung wird von einem geschichteten verhornten Plattenepithel, das dem Bindegewebe glatt aufliegt, gebildet. Als Inhaltsmasse findet sich lamellös geschichtetes, verhorntes Material, sowie zelliger Detritus.

\section{Autoplastische Transplantationen von normalem Epithel.}

\section{(Kontrolltransplantationen.)}

Versuch 1. Exstirpiert nach 8 Tagen. Das herausgenommene Stück erweist sich als eine mit geschichtetem, verhorntem Plattenepithel ausgekleidete 
Cyste. Als Inhalt finden sich nelkrotische Epithelzellen, sowie Haarfragmente. Im umgebenden Bindegewebe viel Hämosiderinpigment.

Versuch 1a. Ein zweiter Versuch, in dem das autoplastisch implantierte Epithelstückchen ebenfalls nach 8 Tagen exstirpiert worden ist, ergibt mikrosliopisch dieselben Verhältnisse.

Versuch 2. Exstirpiert nach 10 Tagen. Mikroskopisch findet sich ein cystischer Hohlraum mit gefältelter Wandung. Die Wandung der Cyste ist gebildet von geschichtetem, verborntem Plattenepitbel. Als Inhalt findet sich lamellös geschichtetes horniges Material.

Versuch 3. Exstirpiert nach 22 T'agen. Das normale Epithel, das zum Versuche verwandt wurde, war vorher in Rohparaffinöl mit 10 proz. Kaninchenfett getaucht worden. Die mikroskopische Untersuchung ergibt mehrere im Bindegewebe gelegene Hohlräume, welche mit geschichtetem und verhorntem Epithel ausgekleidet sind. Das Epithel sitzt dem Bindegewebe glatt auf. Als Inhalt finden sich in allen cystischen Hohlräumen desquamierte Hornmassen und eine grosse Anzahl von Leukozyten, von denen viele Zeichen des Kern- und Protoplasmazerfalles erkennen lassen. In dem Bindegewebe um die Cysten herum finden sich mebrere grosse kugelige, extrahierten Fetttropfen entsprechende Hohlräume. Den Wandungen dieser liegen zahlreiche vielkernige Resorptionsriesenzellen an.

Versuch 4. Exstirpiert nach 26 T'agen. Es findet sich ein klein-erbsengrosses Stück. Bei der mikroskopischen Untersuchung erweist sich das Präparat als eine Plattenepithelcyste. Als Inhaltmassen finden sich konzentrisch geschichtete, mit Leukozyten durchsetzte Hornlamellen, sowie zusammenhängende Lagen von glattem Epithel, letztere jedoch imZustand derDegeneration. Das Epithel der Cyste-verhorntes, gesebichtetes Plattenepithel -- sitzt dem Bindegewebe glatt auf. Nur an einigen Stellen zeigt es plumpe und kugelige, in das Bindegewebe hineinragende Zapfen mit zentralen, konzentrisch geschichteten Hornkugeln, sowie kleinen Talgdrüsen.

Versuch 5. Exstirpiert nach 38 Tagen. Das Präparat weist wiederum eine Plattenepithelcyste auf, deren Inhalt aus desquamierten Hornlamellen besteht. An einzelnen Stellen zeigt die Cystenwand Fältelung. Das Epithel macht einen lebensfrischen Eindruck im histologischen Bilde.

\section{Homoioplastische Transplantationen von gewuchertem Epithel.}

Versuch 1. Rohparaffinölwucherung, exstirpiert nach 8 Tagen. Mikroskopisch findet sich ein mit hornigem, stark von Leukozyten durchsetztem Material ausgefüllter Hohlraum in Bindegewebe. An einer Stelle zeigt die Wandung des Hoblraumes Bekleidung mit einer Lage von mehrschichtigem Plattenepithel. In der Wandung dieses Hohlraums sind mehrere Knoten von Granulationsgewebe mit zahIreichen Riesenzellen, Haarfragmenten, sowie Ilornlamellen zu erkennen.

Versuch 2. Robparaffinölwucherung, exstirpiert nach 14 Tagen. Das Epithel, das vorher 28 Tage autoplastisch transplantiert war, besteht mikroskopisch aus lamellös geschichtetem, hornigem Material, Lagen von in Nekrose befindlichem Plattenepithel, welche in einem von zahlreichen Riesenzellen durchsetzten Bindegewebe gelegen sind.

Versuch 3. Rohparaffinölwucherung, exstirpiert nach 28Tagen. Das Epithel war vorher 10 Tage autoplastisch transplantiert gewachsen. Die mikroskopische Unter- 
suchung des mittelstecknadelkopfgrossen Epithelstückes ergibt nur an einer Stelle als letzten Rest der implantierten Epithelmassen einen Komplex lamellös geschichteten, hornigen Materials. Man erliennt hier an einzelnen Stellen noch undeutlich Konturen einer aussen dem hornigen Material aufliegenden kontinuierlichen Lage ron

Figur 3.

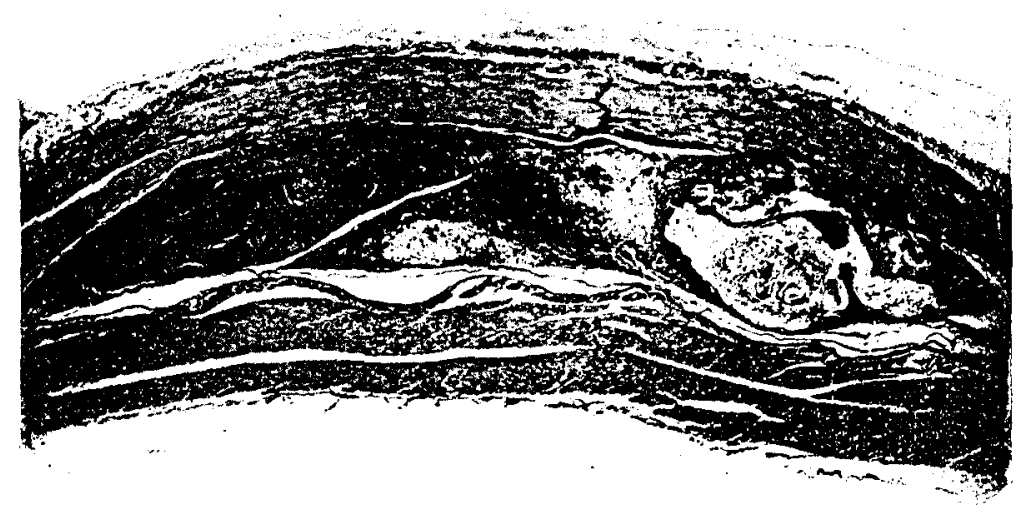

Subkutane homoioplastische Transplantation von gewuchertem Epithel; exstirpiert nach 28 Tagen. Vergrösscrung 26 fach. (Versuch 3.)

Plattenepithel. Dasselbe zeigt Durchwanderung von Leukozyten. Die Hauptmasse des Präparates wird eingenommen von einem zellreichen, besonders lymphozytär durchsetzten Granulationsgewebe mit eingelagerten Gruppen ron Riesenzellen.

\section{Homoioplastische Transplantationen von normalem Epithel.}

(Kontrolltransplantationen.)

Versuch 1. Exstirpiert dem spontan verstorbenen Tiere nach 10 tägigem Wachstum. Mikroskopisch findet sich ein annähernd liugeliger, mit verhorntem Plattenepithel ausgekleideter Hohlraum. Als Inhait findet sich horniges, desquamiertes Material.

Versuch 2. Exstirpiert nach 16 Tagen. Mikrosliopischer Befund wie bei 1.

\section{Intravenöse Transplantationen von gewuchertem Epithel. Autoplastische Transplantationen.}

1. Von gewucbertem Epithel.

2. Von normalem Epithel. (Kontrolltransplantationen.)

Versuch 1. Untersuchung der Lungen nach 17 Tagen.

Versuch 1. Untersuchung der Lungen nach 17 Tagen.

Versuch 2. Untersuchung der Lungen nach 20 l'agen.

Vorsuch 2. Untersuchung der Lungen nach 20 Tagen.

Versuch 3. Untersuchung der Lungen nach 20 Tagen.

Versuch 3. Untersuchung der Lungen nach 25 Tagen.

Versuch 4. Untersuchung der Lungen nach 25 Tagen $\%$. 


\section{Homoioplastische Transplantationen.}

1. Vongewuchertem Epithel.

Versuch 1. Untersuchung der Lungen nach 17 Tagen.

Versuch 2. Untersuchung der Lungen nach 20 Tagen.

Versuch 3. Untersuchung der Lungen nach 20 'Tagen*.

Versuch 4. Untersichung der Lungen nach 70 T'agen.

Versuch 5. Untersuchung der Lungen nach 70 Tagen.

Bei den mit dem * bezeichneten Versuchen wurde in die Vena jugularis externa, bei den übrigen Versuchen in die Randvene des Obres injiziert.

Wie aus der obenstehenden Versuchstabelle hervorgeht, habe ich eine ganze Reihe von Versuchen intravenöser Transplantationen gewucherten Epithels, teils an demselben Tiere, von dem die Wucherung stammte (autoplastisch), teils an anderen Tieren (homoioplastisch) rorgenommen. Zur Kontrolle dienten Parallelversuche von teils autoplastischer, teils homoioplastischer intravenöser Transplantation von normalem Epithel. Die Injektionen erfolgten teils in die Randrene des Ohres, teils in die Vena jugularis externa. Vor der Injektion wurde das Epithel in einem sterilen Mörser zerrieben und die Aufschwemmung desselben in physiologischer Kochsalzlösung mit einer Kanüle von eben noch angängiger Kalibrität injiziert. Die Tiere wurden nach verschiedenen Zeiten getötet und die Lunge einer genauen Inspektion unterworfen. Es gelang aber nie, an den Lungen makroskopische Veränderungen zu finden, welche auf eine Epithelembolie lingedeutet hätten. Ich war somit genötigt, da ich makroskopisch gar keinen Anhaltspunkt hatte, wo die Epithelembolie aufzufinden wäre, möglichst viele aus verschiedenen Teilen des Lungengewebes herausgeschnittene Stuickchen zu untersuchen. Dies ist auch geschehen. Trotz gewissenhafter Untersuchung war ich jedoch nur ein einziges Mal in der Lage, den Befund einer Epithelzellenembolie zu elheben, und zwar handelte es sich um den Versuch Nr. 2 der autoplastischen Transplantationen von normalem Epithel.

Es zeigte sich hier ein arterielles Gefäss von einem halben Millimeter Durchmesser vollständig in seinem Lumen von transplantiertem Material verschlossen, und zwal bestand dieses aus Haulen von Plattenepithel, welche von einer Menge ron einkermigen Zellen rom Charakter grosser Lymphozyten sowie (pseudoeosinophiler) Leukozyten durchsetzt und umgeben waren. Die Epithelzellen zeigten im allgemeinen gute Kern- 
färbung. Der Kern einiger war jedoch blass, schattenhaft. Das Protoplasma aller war vakuolisiert, und von wabigem Bau. Zeichen stärkerer Degeneration an den Epithelzellen konnte man an den Partien erkennen, wo die Haufen der Epithelzellen stark von Leukozyten durchsetzt waren. Hier waren Erscheinungen von Karyolyse und Plasmolyse deutlich sichtbar. $\mathrm{Da}$ es nicht angängig war, die Lungen sämtlicher zu den intravenösen Transplantationensversuchen verwandter Tiere in lückenlose Serien zu zerlegen, bin ich nicht in der Lage, über das Verhalten des bei den Versuchen doch sicher in die Lungen embolisierten gewucherten Epithels etwas auszusagen, und es haben so meine Versuche mit der intravenösen Transplantation gewucherten Epithels zu keinem definitiven Resultat geführt. Jedoch glaube jch mich berechtigt, anzunehmen, dass ein irgendwie vom Normalen, bisher Bekannten abweichendes Verhalten des intravenös injizierten und sekundir in die Lunge gelangten atypischen Epithels nicht statt hatte. Denn sonst wäre ich wohl in der Lage gewesen, bei meinen überaus zahlreichen Schnitten in einem oder dem anderen Präparate dies beobachten zu können. Dass ich nie in der Lage war, die in die Lunge embolisierten Epithelien in den mikroskopischen Schnitten zu finden, legt den Gedanken nahe, dass die embolisierten Epithelzellen bald in der Lunge zur Degeneration gekommen sind.

Unterwirft man die sublutanen, teils autoplastischen, teils homoioplastischen Transplantationsversuche der experimentell erzeugten atypischen Epithelwucherungen nochmals einem kurzen Ueberblick, so hat sich bei diesen nicht feststellen lassen, dass das atypisch gewucherte Epithel sich irgendwie anders verhält, wie normales Epithel. Was gefunden wurde, waren mit Epithel ausgekleidete Cysten in der Art, wie sie bei Verlagerungen von Epithel so häufig beobachtet werden. Die einzige Beobachtung aus meinen Versuchen, welche für die Frage der Transplantation überhaupt ein gewisses Interesse beanspruchen dürfte, war die, dass das homoioplastisch transplintierte Epithel früher der Degeneration anheim fällt, wie das autoplastisch transplantierte, ein Befund, wie er mit den Erfahrungen über homoioplastische Transplantation aus neuester Zeit übereinstimmt. 